\title{
Routing in Mobile Ad-Hoc Networks using Social Tie Strengths and Mobility Plans
}

\author{
Riten Gupta*, Niyant Krishnamurthi*, Uen-Tao Wang ${ }^{\dagger}$, Tejaswi Tamminedi*, and Mario Gerla ${ }^{\dagger}$ \\ *UtopiaCompression Corp., Los Angeles, CA \\ \{riten, niyant\}@utopiacompression.com \\ $\dagger$ UCLA, Los Angeles, CA
}

\begin{abstract}
We consider the problem of routing in a mobile ad-hoc network (MANET) for which the planned mobilities of the nodes are partially known a priori and the nodes travel in groups. This situation arises commonly in military and emergency response scenarios. Optimal routes are computed using the most reliable path principle in which the negative logarithm of a node pair's adjacency probability is used as a link weight metric. This probability is estimated using the mobility plan as well as dynamic information captured by table exchanges, including a measure of the social tie strength between nodes. The latter information is useful when nodes deviate from their plans or when the plans are inaccurate. We compare the proposed routing algorithm with the commonly-used optimized link state routing (OLSR) protocol in ns-3 simulations. As the OLSR protocol does not exploit the mobility plans, it relies on link state determination which suffers with increasing mobility. Our simulations show considerably better throughput performance with the proposed approach as compared with OLSR at the expense of increased overhead. However, in the high-throughput regime, the proposed approach outperforms OLSR in terms of both throughput and overhead.
\end{abstract}

\section{INTRODUCTION}

Mobile ad-hoc networks (MANETs) have been studied for several decades and, with recent advances in wireless communication technology, are expected to find increasing use in a wide range of applications. In general a MANET must be designed to accommodate arbitrary mobility of the constituent nodes (under reasonable speed constraints). The nodes may or may not move in groups, and their trajectories are generally unknown. Many MANET routing algorithms and protocols, both proactive and reactive, have been proposed over the years [1], some of which take advantage of known characteristics of the underlying network, and others which operate in the most general case. For example, the LANMAR protocol [2] is specifically designed for networks with group mobility, while Optimized Link State Routing (OLSR) [3], makes no assumptions on mobility. Many more such examples of environment-specific protocols exist. See [1] for a summary. In the last decade, researchers have shown that routing in delay-tolerant networks (DTNs) can be aided by dynamically gathered information about the social graph [4] of the underlying network [5] [6]. In particular, a node's centrality, which in a social sense expresses its relative importance, can be used to determine its suitability for forwarding packets.

In this paper, we examine MANETs formed by a set of nodes participating in a collaborative activity for a finite time duration. An example is a military setting in which the nodes consist of mounted and dismounted soldiers carrying out a carefully planned mission. The mission plan includes the trajectory of each node, specified by waypoints, and nodes travel in groups. The trajectory information is useful for predicting node pair adjacencies and the group nature of the node travel gives rise to a novel adjacency prediction method using social tie strengths, which is beneficial when nodes deviate from their planned trajectories. This type of planned network, referred to as a tactical edge network, also arises in emergency response; for example, in disaster relief missions. A network of unmanned aerial vehicles (UAVs) with known flight plans is another potential example. Previous studies [7] [8] showed good results in using a "social tie centrality" metric for packet forwarding in information-centric networks. In this work, we derive a novel routing algorithm for tactical edge networks which exploits mobility plans and disseminates social tie information in a manner similar to [7]. Both pieces of information are used to make routing decisions by the algorithm, which we call Tactical Edge Network Social Routing (TENSR). We compare the performance of this algorithm in terms of throughput, delay, and overhead, to OLSR for several representative cases We find that significant throughput improvement relative to OLSR can be obtained by exploiting the mobility plan and social information.

The remainder of this paper is organized as follows. In Section II we introduce the tactical edge networks that are the target applications for TENSR. The routing algorithm is then described in detail in Section III We present ns-3 simulation studies in Section IV Finally, Section V concludes the paper.

\section{TACTICAL Edge Networks}

In this section we describe the characteristics of a tactical edge MANET both qualitatively and mathematically. We also discuss how these features can be used to aid routing decisions. We assume that $N$ network nodes carry out a mission that spans $T$ seconds.

\section{A. Mobility Plans}

In a tactical edge MANET each node is associated with a mobility plan, which consists of a finite set of waypoints describing the node's planned trajectory throughout a mission. In most cases, the nodes travel in groups as they carry out the mission. The nodes' actual trajectories are expected to deviate 
somewhat from their mobility plans. In some cases, such as unplanned emergency events, a node may drastically change its trajectory. In others cases deviations will be much smaller and can be modeled by a random jitter process. Mobility plans represent a very useful set of a priori information that can facilitate routing decisions.

For node $i$, the mobility plan consists of a list of pairs $\left(t_{1}, \theta_{i}\left(t_{1}\right)\right), \ldots,\left(t_{n}, \theta_{i}\left(t_{n}\right)\right)$ where $n$ is the number of waypoints in node $i$ 's mobility plan, $t_{j}$ is the time of the $j$ th waypoint, and $\theta_{i}\left(t_{j}\right) \in \mathbb{R}^{2}$ is a 2-dimensional Euclidean position vector. 1 The list of waypoints is considered a discrete sampling of a node's trajectory. Between waypoints, the node is assumed to travel at constant velocity along the line segment connecting the two points. Thus, for each node a complete planned trajectory exists: $\left\{\theta_{i}(t): t \in[0, T]\right\}$.

\section{B. Position Location Information}

In military and emergency response networks, position location information (PLI) may be available, for example from a blue force tracking system. Such a system communicates position information about each node to all nodes. In this study, we assume that PLI is disseminated on a channel independent of the network traffic. This information may be inaccurate (due to errors in the geolocation mechanism) and unreliable (due to latencies or congestion in the dissemination protocol). Let $\phi_{i}(t) \in \mathbb{R}^{2}$ be the PLI estimate of node $i$ 's location at time $t$.

\section{Social Tie in Tactical Edge Networks}

As we discuss in Section III-C in a MANET with uncertain link state, we become interested in the probability that a pair of nodes is adjacent-that is, whether a link between the nodes exists in the communication graph-for this information proves useful in route selection. If we define a social graph consisting of the same vertices (nodes) as the communication graph, with edge weights corresponding to the social tie strengths between pairs of nodes, then this graph can be useful in estimating the probability of adjacency in the communication graph.

Tie strengths and their implications for social networks were proposed in [9] and extended in [10], while the application of tie strengths to MANET routing is analyzed in [11]. Several factors are important in defining a social tie metric, including intimacy/closeness, frequency of contacts, reciprocity, and recency. In [7], a social tie metric based on frequency and recency was shown to be effective in routing for delay-tolerant MANETs. In our algorithm, we use a simple social tie definition which takes into account only the frequency of encounters, as this lends itself to a simple adjacency probability estimator. Specifically, we define the social tie $R_{i, j}(t)$ between nodes $i$ and $j$ at time $t$ to be the number of encounters between these two nodes in the last $T_{m e m}$ seconds. The parameter $T_{m e m}$ is called the social tie memory. Here an encounter refers to the nodes being adjacent at some point during a measurement

\footnotetext{
${ }^{1}$ We focus on 2-D mobility in this paper but point out that the analysis can be extended to the 3-D case in a straightforward manner.
}

interval $T_{\text {int }}{ }^{2}$ Letting $R_{m e m}=T_{m e m} / T_{\text {int }}$ (assumed an integer), we see that $R_{i, j}(t) \leq R_{m e m}$ and that the probability of adjacency between nodes $i$ and $j$ can be estimated as

$$
P(i, j \text { adjacent }) \approx \frac{R_{i, j}(t)}{R_{m e m}} .
$$

\section{Routing Algorithm}

Here we describe the routing algorithm. The algorithm consists of two main activities: table exchanges and route selection. The goal of the table exchange process is to supply each node with a sufficent amount of information from which it can estimate node adjacency probabilities. These probabilities are then used to determine most reliable paths.

\section{A. Link State Data}

We first describe the various data structures used by the nodes to exchange information and estimate adjacency probabilities. Let $q$ be the index of an arbitrary node in the network.

1) Social Tie: Node $q$ stores an $N \times N$ matrix $\mathbf{R}^{(q)}$ of estimated social ties between each node pair ${ }^{3}$ Associated with each entry in this matrix is a timestamp indicating when the value was determined. These timestamps are stored in matrix $\mathbf{T}_{S T}^{(q)}$. These matrices are updated dynamically throughout the mission.

2) Empirical Adjacency: While the social tie measures the number of encounters, the empirical adjacency of a pair of nodes indicates whether or not the nodes are currently adjacent. These empirical adjacencies are stored in matrix $\mathbf{A}^{(q)}$ and their corresponding timestamps, indicating the time at which the adjacency (or non-adjacency) was determined, are stored in matrix $\mathbf{T}_{A d j}^{(q)}$. They are updated dynamically

3) Pairwise Distance: Node $q$ has a pairwise-distance matrix $\mathbf{D}^{(q)}$. The $(i, j)$ th element of this matrix is the measured geographic distance between nodes $i$ and $j$. The corresponding timestamp matrix is $\mathbf{T}_{D}^{(q)}$. These matrices are updated dynamically during node encounters.

4) Mobility Plans: Node $q$ has the complete list of waypoints for each node. Thus, as described in Section II-A node $q$ has knowledge of $\theta_{i}(t)$ for all $i \in\{1, \ldots, N\}$ and $t \in[0, T]$. This information is known a priori and is static (i.e., not updated dynamically).

5) PLI: At any time $t$, node $q$ has an estimate of each other node's (say node $i$ 's) position in the form of PLI $\phi_{i}^{(q)}(t)$. The accuracy of this information depends in large part on its staleness. Thus node $q$ also stores the timestamp for each other node's PLI: $T_{p, i}^{(q)}$.

6) Other Parameters: Several other parameters needed by the routing algorithm are described here. The variance of each node's (say node $i$ 's) trajectory $\sigma_{n, i}^{2}$ is known by all nodes. The variance of the PLI estimate is also known, but since this depends on staleness, the nodes store a set of breakpoints defining a piecewise linear function representing

\footnotetext{
${ }^{2}$ This definition of social tie is a special case of the one in [7], with $f(x)$ the indicator function of the interval $\left[0, T_{m e m}\right]$.

${ }^{3}$ All matrices described here are symmetric and only the upper or lower triangular parts must be stored
} 
the PLI variance versus the staleness, denoted $\sigma_{p}^{2}(\tau)$ where $\tau$ is the staleness. Each node thus has knowledge of the function $\sigma_{p}^{2}(\tau)$. The maximum communication range of each node $d_{\max , i}$ is known by all nodes, as is the maximum velocity $v_{\max , i}$.

\section{B. Information Exchange}

Location information from a priori mission trajectories and PLI is an invaluable aid for routing. However, nodes may deviate from their planned trajectories and PLI may be inaccurate or stale. Thus, dynamic information is also used to make routing decisions. Specifically, a node pair's adjacency, distance, and social tie are used. In order for nodes to get this information, a dissemination method is employed consisting of handshaking and table exchanges. Although the purpose of this dissemination method is to give the nodes the information necessary for routing, it must also minimize communications overhead to be useful. In this section, we briefly describe this information dissemination process. Please refer to [12] for more detail.

Information exchanges are done using two types of messages: HELLO messages and INFO messages. A HELLO message is a small message sent periodically which is used to identify neighbors. A HELLO message also conveys small pieces of information to the recipient. An INFO message is used to transmit table information from one node to another. This information is either adjacency, distance, or social tie. The average time interval between emissions of HELLO messages by a node is called the HELLO interval. This interval is a design parameter. A low HELLO interval results in greater information sharing, leading to better route selection and improved throughput. However, this also entails a larger communication overhead as many HELLO messages are transmitted per unit of time. Similarly, the INFO interval is the minimum time duration between emissions of INFO messages by a node. This interval is also a design parameter. The tradeoffs described for the HELLO interval apply to the INFO interval as well.

Table exchanges are done during node encounters. During an encounter, a pair of nodes determines the geographic distance between the pair. It is assumed that the nodes have the necessary processing hardware to make this estimate. The nodes also update their social tie and adjacency information and decide what information to transmit to one another. In [12], the table exchange process, along with a description of the message formats, is given. Note that prior knowledge of social ties can be used to initialize the nodes' social tie matrices, but in general the social structure is considered a dynamic process which is tracked throughout the mission.

\section{Route Selection}

Since in a tactical edge network, link adjacencies are usually not known with certainty, routes may not be chosen based on typical shortest path principles. Instead, we choose paths that are most reliable [13]. Let $\mathcal{S}_{m, n}$ be the set of all paths between nodes $m$ and $n$ in the complete graph. Each such path $s$ is a set of links which may be represented as node pairs. So for example, if the link between nodes $i$ and $j$ is in path $s$ then $(i, j) \in s$. The most reliable path between $m$ and $n$ is

$$
\begin{aligned}
s_{m, n}^{*} & =\underset{s \in \mathcal{S}_{m, n}}{\operatorname{argmax}} \prod_{(i, j) \in s} \operatorname{Pr}(i, j \text { adjacent }) \\
& \approx \underset{s \in \mathcal{S}_{m, n}}{\operatorname{argmax}} \prod_{(i, j) \in s} \hat{p}_{i, j} \\
& =\underset{s \in \mathcal{S}_{m, n}}{\operatorname{argmin}} \sum_{(i, j) \in s}-\log \hat{p}_{i, j}
\end{aligned}
$$

where $\hat{p}_{i, j}$ is an estimate of $\operatorname{Pr}(i, j$ adjacent $)$. Here we are assuming that the link adjacency probabilities are independent. From (2), optimal routes can be found using Dijkstra's algorithm on a graph with edge weights given by $-\log \hat{p}_{i, j}$ for edge $(i, j)$.

\section{Link Adjacency Probability Estimation}

Most reliable path (MRP) route selection requires that each node has an estimate of adjacency probability for each node pair in the network. The adjacency probabilities can be estimated in a variety of ways, depending on what information is available to the estimating node. These methods are described in this section. In the following we assume that node $q$ is estimating the probability of adjacency of nodes $i$ and $j$, with $i \neq j$, (but $q$ may or may not equal $i$ or $j$ ).

1) Case 1: $q \in\{i, j\}:$ If $q$ is one of the nodes in the pair for which adjacency probability is needed, then the most accurate estimate can be found using node $q$ 's adjacency matrix. The handshaking mechanism ensures that each node always knows its neighbor list accurately [12]. Therefore, we set $\hat{p}_{i, j}=\mathbf{A}_{i, j}^{(q)}$.

2) Case 2: Using Previous Adjacency Information: Assuming $q \notin\{i, j\}$, in some cases the adjacency of nodes $i$ and $j$ can still be confirmed with certainty. Suppose $\mathbf{A}_{i, j}^{(q)}=1$ and let $\tau=t-\mathbf{T}_{D, i, j}^{(q)}$ where $t$ is the current time. Thus $\tau$ is the staleness of the pairwise distance estimate $d=\mathbf{D}_{i, j}^{(q)}$. Then if

$$
\tau \leq \tau_{\text {thresh }}=\frac{d_{\max }-d}{v_{\max , i}+v_{\max , j}}
$$

(where $d_{\max }=\min \left(d_{\max , i}, d_{\max , j}\right)$ ), then the nodes must currently be adjacent. Therefore, if $\tau \leq \tau_{\text {thresh }}$, then we set $\hat{p}_{i, j}=1$.

3) Case 3: Using Location Information: Both a priori trajectory plans and PLI help to determine adjacencies. Actual trajectories differ from the waypoint specification due to (small) jitter processes that occur even when the nodes follow their mission plans, but also due to emergencies and changes of plans which cause large differences with respect to the waypoints. PLI is known to be noisy due to latency and error in geolocation.

Gaussian Models: We model the deviation between trajectories and trajectory plans (based on waypoints) as Gaussian with known covariance $\sigma_{n, i}^{2} I$. Similarly, the PLI error is modeled as Gaussian with staleness-dependent covariance $\sigma_{p, i}^{2}(\tau) \cdot I$. In general, it is expected that PLI error variances are larger than a priori error variances. In the case of nodes that 
deviate from the mission plan due to emergencies or other unplanned events, the a priori trajectory provides no information regarding the true location. Our approach is to first determine if a node has deviated significantly from its plan using a simple hypothesis test based on observation of PLI. If such a deviation has not occurred, then the node's location $X_{i}(t)$ is modeled as Gaussian with mean equal to the planned location $\theta_{i}(t)$ and covariance $\sigma_{n, i}^{2}$, or $X_{i}(t) \sim \mathcal{N}\left(\theta_{i}(t), \sigma_{n, i}^{2} \cdot I\right)$. If a deviation has occurred, then the location is modeled as Gaussian with mean equal to the PLI $\phi_{i}(t)$, and covariance $\sigma_{p, i}^{2}(\tau) \cdot I$, or $X_{i}(t) \sim \mathcal{N}\left(\phi_{i}(t), \sigma_{p, i}^{2}(\tau) \cdot I\right)$.

Hypothesis Test: Dispensing with time dependencies in the notation for now, we model the PLI as $\phi_{i}=X_{i}+M_{i}$ where $M_{i} \sim \mathcal{N}\left(0, \sigma_{p, i}^{2} \cdot I\right)$. Next, let $\mathcal{H}_{0}$ be the hypothesis that node $i$ is travelling according to its plan, or

$$
\mathcal{H}_{0}: X_{i}=\theta_{i}+N_{i}
$$

with $N_{i} \sim \mathcal{N}\left(0, \sigma_{n, i}^{2} \cdot I\right)$. Then $\mathcal{H}_{0}$ can be written

$$
\mathcal{H}_{0}: \phi_{i} \sim \mathcal{N}\left(\theta_{i}, \sigma^{2} I\right)
$$

where $\sigma^{2}=\sigma_{p, i}^{2}+\sigma_{n, i}^{2}$. Next, note that

$$
\frac{\left\|\phi_{i}-\theta_{i}\right\|^{2}}{\sigma^{2}} \sim \chi_{2}^{2}
$$

Let $1-\alpha$ be a confidence level, where $0<\alpha \ll 1$ and set $\operatorname{Pr}\left(\left\|\phi_{i}-\theta_{i}\right\| \leq T \mid \mathcal{H}_{0}\right)=1-\alpha$, with $T$ a threshold. Solving for $T, \mathcal{H}_{0}$ should be rejected whenever

$$
\left\|\phi_{i}-\theta_{i}\right\|>\sigma \sqrt{F_{\chi_{2}^{2}}^{-1}(1-\alpha)}
$$

where $F_{\chi_{2}^{2}}$ is the cumulative distribution function (CDF) of a chi square variate with two degrees of freedom.

Location-Based Adjacency Probability Estimation: The hypothesis test is conducted for both node $i$ and $j$. For node $i$, if $\mathcal{H}_{0}$ is rejected, then the location is modeled as

$$
X_{i}(t) \sim \mathcal{N}\left(\phi_{i}(t), \sigma_{p, i}^{2}(\tau) \cdot I\right) .
$$

Otherwise it is modeled as

$$
X_{i}(t) \sim \mathcal{N}\left(\theta_{i}(t), \sigma_{n, i}^{2} \cdot I\right)
$$

For node $j$, a similar model is obtained for $X_{j}(t)$. Thus let $\left(\mu_{i}, \sigma_{i}^{2} I\right)$ and $\left(\mu_{j}, \sigma_{j}^{2} I\right)$ be the means and covariances of nodes $i$ and $j$ respectively. Then

$$
\frac{\left\|X_{i}(t)-X_{j}(t)\right\|^{2}}{\sigma_{i}^{2}+\sigma_{j}^{2}} \sim \chi_{2}^{2}(\lambda) .
$$

The non-centrality parameter of this chi-square random variable is $\lambda=\left\|\mu_{i}-\mu_{j}\right\|^{2} /\left(\sigma_{i}^{2}+\sigma_{j}^{2}\right)$ and the probability of adjacency of nodes $i$ and $j$ is

$$
\hat{p}_{i, j}=\operatorname{Pr}\left(\left\|X_{i}(t)-X_{j}(t)\right\| \leq d_{\max }\right)=F_{\chi_{2}^{2}(\lambda)}\left(\frac{d_{\max }^{2}}{\sigma_{i}^{2}+\sigma_{j}^{2}}\right)
$$

where $F_{\chi_{2}^{2}(\lambda)}$ is the non-central chi-square CDF with noncentrality parameter $\lambda$.
4) Case 4: Using Social Tie: Next, if PLI information is unavailable for one or both nodes $i, j$ (i.e., it is so stale that we consider it useless), then we use the social tie between the nodes to estimate the adjacency probability. Recall from equation (1) that the adjacency probability estimate is

$$
\hat{p}_{i, j}=\frac{R_{i, j}(t)}{R_{m e m}} .
$$

This equation is used when the staleness of either node's PLI is greater than a threshold, called the PLI staleness threshold.

5) Case 5: Default Case: Finally, if the social tie is also deemed too stale to be useful, a default non-zero adjacency probability is assigned: $\hat{p}_{i, j}=p_{0}$. Setting $p_{0}$ to 0 would preclude the consideration of link $(i, j)$ in the MRP routing process. On the other hand, setting $p_{0}$ too high will cause MRP to favor the link, which may not exist. Thus a small positive value is preferred for $p_{0}$. This value is used when the social tie staleness of nodes $i, j$ is greater than a threshold, called the social tie staleness threshold.

\section{Simulation Results}

In this section, we present the results of several simulations comparing the TENSR routing protocol to OLSR for various tactical edge networks. The simulations are done using ns-3 [14]. The nodes follow mobility plans specified by waypoints. These waypoints are known to the routing protocol (they are part of the a priori information). However, only the waypoints corresponding to the first half of the mission duration, i.e., $[0, T / 2]$, are known. Beyond this, the mobility is unknown to the routing protocol.

\section{A. Models and Parameters}

1) Node Mobility: Nodes travel at constant velocity between waypoints, which are generated randomly based on desired node velocities. A Gaussian jitter process with covariance $\sigma_{n}^{2} \cdot I$ is added to the linear movement. Nodes travel in groups and all nodes within a group share the same mobility plan (waypoints), but have independent jitters. We consider the following groupings: 7 groups with 3 nodes per group, 5 groups with 4 nodes per group, and a 10 groups of 2 nodes. A geographical area with dimensions $1500 \times 1500$ square meters is considered. Typically, tactical edge networks are connected; the missions are designed as such. Therefore, when generating waypoints randomly, we ensure a high likelihood of connectedness, or equivalently a low average network partitioning (ANP) [15].

2) PLI Model: PLI comes from a channel independent of the communication network; for example, from a blue force tracking system. The PLI is sporadic, and not all nodes in the network obtain the PLI at every PLI transmission. PLI is implemented in simulation as an oracle residing on each node, which informs a random subset of all other nodes of its position (with an additive noise component). The following parameters characterize the PLI model. First the broadcast interval is the time interval at which PLI broadcasts are sent. Additionally, a broadcast interval jitter is added to this interval 
TABLE I

SIMULATION PARAMETERS

\begin{tabular}{l|l}
\hline Parameter & Values simulated \\
\hline \hline Groups, nodes/group & $(2,10),(3,7),(4,5)$ \\
\hline Simulation duration, $T$ & $10 \mathrm{~min}$ \\
\hline Deviation time $(T / 2)$ & $5 \mathrm{~min}$ \\
\hline Node velocity & $10 \mathrm{~m} / \mathrm{s}, 20 \mathrm{~m} / \mathrm{s}, 30 \mathrm{~m} / \mathrm{s}$ \\
\hline Jitter standard deviation, $\sigma_{n}$ & $10 \mathrm{~m}($ lat and lon) \\
\hline Radio range & $500 \mathrm{~m}$ \\
\hline Geographic area & $1500 \times 1500$ square meters \\
\hline \# Monte-Carlo trials & 30 \\
\hline HELLO interval & $0.5 \mathrm{sec}$ \\
\hline INFO interval & $4 \mathrm{sec}$ \\
\hline Social tie memory, $R_{m e m}$ & 10 \\
\hline Social tie staleness threshold & $60 \mathrm{sec}$ \\
\hline PLI broadcast interval & $5 \mathrm{sec}$ \\
\hline PLI broadcast interval jitter & $5 \mathrm{sec}$ \\
\hline PLI broadcast reach probability & 0.5 \\
\hline PLI broadcast delay & $3 \mathrm{sec}$ \\
\hline PLI standard deviation, $\sigma_{p}^{2}(\tau)$ & $10 \mathrm{~m}$ (lat and lon) at $\tau=0$ \\
\hline & $20 \mathrm{~m}$ (lat and lon) at $\tau=20 \mathrm{sec}$ \\
\hline & $30 \mathrm{~m}$ (lat and lon) at $\tau=30 \mathrm{sec}$ \\
\hline PLI staleness threshold & $10 \mathrm{sec}$ \\
\hline Hypothesis test confidence level & $95 \%$ \\
\hline OLSR HELLO interval & $2 \mathrm{sec}$ \\
\hline OLSR TC interval & $5 \mathrm{sec}$
\end{tabular}

randomly. The broadcast reach probability is the probability of successful PLI reception by any node. Finally the broadcast delay is the delay of the PLI broadcast signal.

3) Traffic Types: We simulated constant bit rate (CBR) traffic and used a simple echo application in which a source node sends a packet to a destination, which then replies to the source, only if and when it receives the original packet. Six CBR flows are used with packet size 1024 bits and rate 1 packet/sec. The sources and destinations are always chosen to lie in separate groups.

4) Summary of Parameters: Table I summarizes the simulation parameters.

\section{B. Comparison with OLSR}

We first compare the TENSR protocol with OLSR in its standard configuration. The standard OLSR configuration uses a HELLO interval of $2 \mathrm{sec}$ and a topology control (TC) interval of $5 \mathrm{sec}$ [3]. As these intervals are somewhat analagous to TENSR's HELLO interval and INFO interval, and the TENSR intervals are shorter than their OLSR counterparts, the results are expected to favor TENSR. We will see that this is the case. Later, we discuss simulations in which both protocols' intervals are approximately the same.

Figure 1 shows the average number of received packets over the course of $10 \mathrm{~min}$ for both protocols for various node velocities and various node groupings with CBR traffic. (See Table I) We found that 30 Monte-Carlo trials were enough to sufficiently estimate the mean. The results correspond to the following TENSR parameters: HELLO inerval $=0.5 \mathrm{sec}$, INFO interval $=4 \mathrm{sec}$. The simulation duration is $10 \mathrm{~min}$, and at $5 \mathrm{~min}$, the nodes deviate from their planned mobility pattern. In all cases, the TENSR throughput is superior to that of (standard) OLSR.

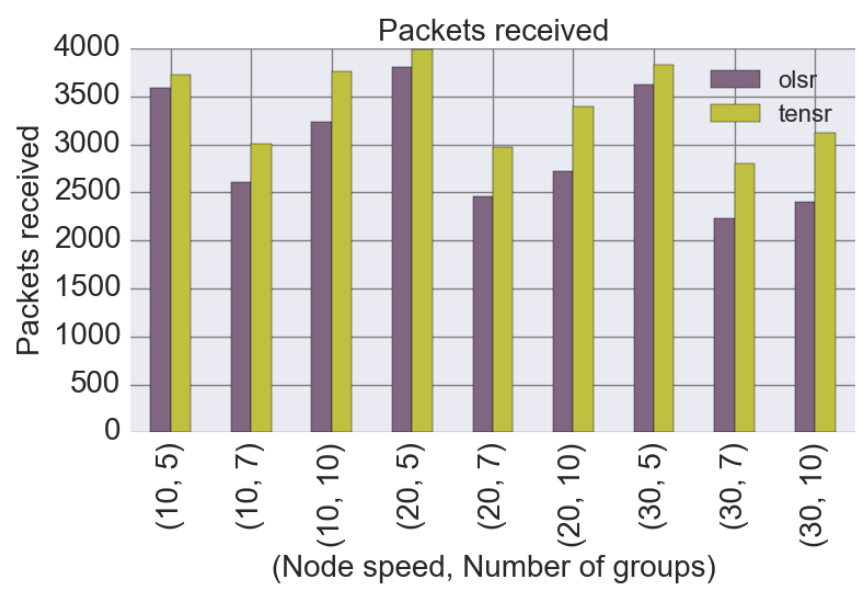

Fig. 1. Average number of packets successfully received with TENSR and OLSR

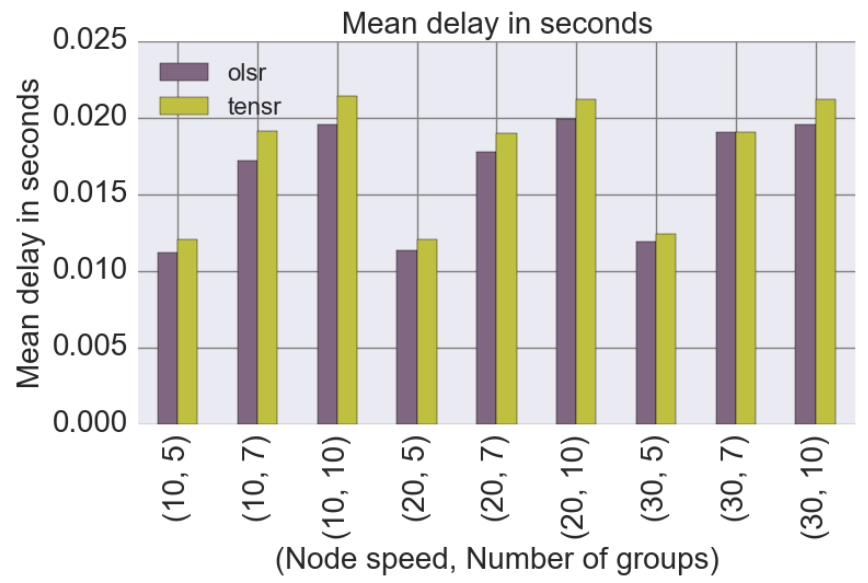

Fig. 2. Average packet delay with TENSR and OLSR

Next, Figure 2 shows the average packet delay for the two protocols, as well as over all packets successfully received) for CBR traffic. Note that the TENSR delay is higher than the corresponding OLSR delays. While this is true in an average sense, a closer inspection shows that in fact packets that are successfully transmitted in both cases (TENSR and OLSR) receive approximately the same delay, whereas packets that are successfully transmitted by TENSR, but not OLSR receive a higher delay. Since these packets do not impact the OLSR average delay, the TENSR average delay is higher. Thus, more packets are transmitted when using TENSR, but these "extra" packets experience a higher-than-average delay. Other packets experience approximately the same delay with both protocols.

\section{Throughput vs. Overhead Comparison}

The results of the previous section show that the proposed routing protocol can exploit the mission and social information to deliver performance superior to OLSR. However, this performance improvement comes at the expense of increased communication overhead in the form of table 


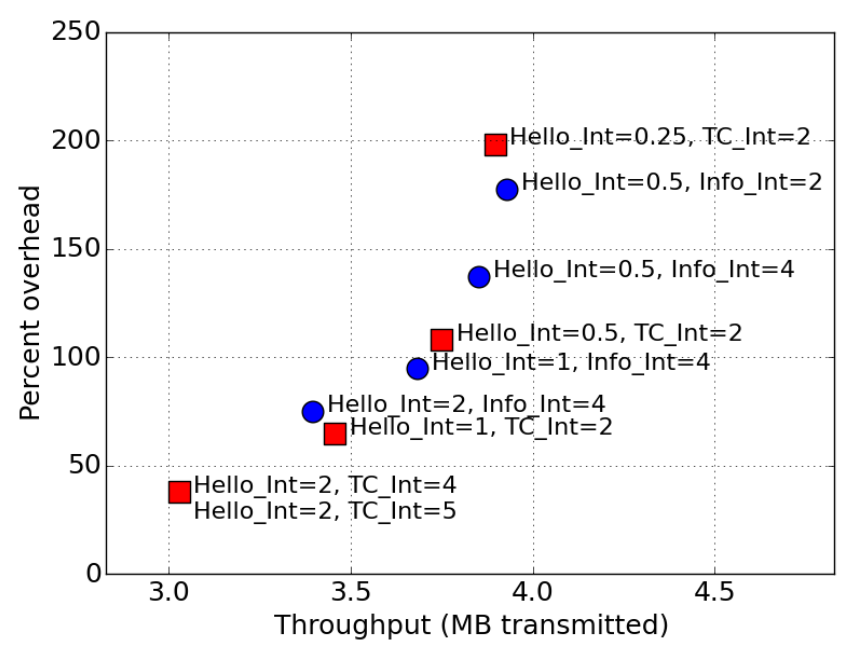

Fig. 3. Throughput vs. overhead. Circles are TENSR simulations, squares are OLSR simulations.

exchange packets. The performance of OLSR can be improved by changing its HELLO interval and topology control interval [16]. In this section, we focus on a single network configuration and run simulations with varying OLSR parameters. We also run TENSR simulations with varying parameters. We use an expanded HELLO message format [12, Sec 3.4.3] which includes some link-state information in the HELLO message and which generally improves performance. We log the number of overhead bits and compare the percent overhead versus throughput for both protocols. These simulations use the following parameters: 3 groups, 7 nodes per group, 10 min simulation time, without deviation from planned mobility, and $20 \mathrm{~m} / \mathrm{s}$ velocity, The HELLO interval, INFO interval, OLSR hello interval, and OLSR TC interval are varied. The remaining parameters are identical to those in Table II

Figure 3 shows the throughput versus percent overhead for both OLSR and the TENSR for various parameter sets. Generally, the two protocols are comparable and tend to lie on the same curve. As the HELLO, INFO, and TC intervals become small, the throughput reaches a limit. In this regime, the proposed protocol provides slightly higher throughput than OLSR for less overhead (approximately 10\%). Thus, we conclude that while the mission and social information is certainly helpful for routing, in some cases, its benefits can be gained by using OLSR with more messaging (and overhead). In the high-throughput regime, however, the proposed protocol is superior to OLSR, even with increased OLSR messaging.

\section{COnCLUSions}

We have developed a novel routing protocol for tactical edge networks which exploits known mission information such as mobility plans, as well as dynamic social information and position location information. The protocol has been compared to OLSR and shown to be superior in terms of throughput and approximately equal in terms of delay, at the expense of increased overhead. In the high-throughput regime, which is achived by reduction of the HELLO and information exchange intervals in both protocols, the TENSR protocol is superior to OLSR in both throughput and overhead.

\section{ACKNOWLEDGEMENTS}

The authors wish to thank Dr. You Lu from UCLA and Dr. Jacob Yadegar from UtopiaCompression Corporation for helpful insights. This material is based upon work supported by the RDECOM-CERDEC-STG-TW under Contract No. W56KGU-15-C-0069.

\section{REFERENCES}

[1] A.-S. K. Pathan and C. S. Hong, "Routing in mobile ad hoc networks," in Guide to Wireless Ad Hoc Networks. Springer, 2009, pp. 59-96.

[2] G. Pei, M. Gerla, and X. Hong, "LANMAR: Landmark routing for large scale wireless ad hoc networks with group mobility," in Proceedings of the 1st ACM International Symposium on Mobile Ad Hoc Networking \& Computing. IEEE Press, 2000, pp. 11-18.

[3] T. Clausen and P. Jacquet, "Optimized link state routing protocol (OLSR)," Internet Requests for Comments, RFC Editor, RFC 3626, October 2003, http://www.rfc-editor.org/rfc/rfc3626.txt [Online]. Available: http://www.rfc-editor.org/rfc/rfc3626.txt

[4] D. Easley and J. Kleinberg, Networks, Crowds, and Markets: Reasoning about a Highly Connected World. Cambridge University Press, 2010.

[5] E. M. Daly and M. Haahr, "Social network analysis for routing in disconnected delay-tolerant MANETs," in Proceedings of the 8th ACM International Symposium on Mobile Ad Hoc Networking and Computing. ACM, 2007, pp. 32-40.

[6] P. Hui, J. Crowcroft, and E. Yoneki, "Bubble rap: Social-based forwarding in delay-tolerant networks," Mobile Computing, IEEE Transactions on, vol. 10, no. 11, pp. 1576-1589, 2011.

[7] Y. Lu, X. Li, Y.-T. Yu, and M. Gerla, "Information-centric delay-tolerant mobile ad-hoc networks," in Computer Communications Workshops (INFOCOM WKSHPS), 2014 IEEE Conference on. IEEE, 2014, pp. $428-433$.

[8] T. Le, Y. Lu, and M. Gerla, "Social caching and content retrieval in disruption tolerant networks (DTNs)," in Computing, Networking and Communications (ICNC), 2015 International Conference on. IEEE, 2015, pp. 905-910.

[9] M. S. Granovetter, "The strength of weak ties," American Journal of Sociology, pp. 1360-1380, 1973.

[10] P. V. Marsden and K. E. Campbell, "Measuring tie strength," Social Forces, vol. 63, no. 2, pp. 482-501, 1984.

[11] E. M. Daly and M. Haahr, "Social network analysis for information flow in disconnected delay-tolerant MANETs," IEEE Transactions on Mobile Computing, vol. 8, no. 5, p. 606, 2009.

[12] U.-T. Wang, "Tactical edge network social routing (TENSR)," Department of Computer Science, UCLA, Los Angeles, CA, Tech. Rep. No. 160006, June 2016.

[13] R. A. Guérin and A. Orda, "QoS routing in networks with inaccurate information: theory and algorithms," IEEE/ACM Transactions on Networking (TON), vol. 7, no. 3, pp. 350-364, 1999.

[14] T. R. Henderson, M. Lacage, G. F. Riley, C. Dowell, and J. Kopena, "Network simulations with the ns-3 simulator," SIGCOMM demonstration, vol. 15, p. 17, 2008.

[15] S. Kurkowski, T. Camp, and W. Navidi, "Two standards for rigorous MANET routing protocol evaluation," in 2006 IEEE International Conference on Mobile Ad Hoc and Sensor Systems. IEEE, 2006, pp. 256-266.

[16] Y. Huang, S. N. Bhatti, and D. Parker, "Tuning OLSR," in 2006 IEEE 17th International Symposium on Personal, Indoor and Mobile Radio Communications. IEEE, 2006, pp. 1-5. 